\title{
A 3D Image Reconstruction Model for Long Tunnel Geological Estimation
}

\author{
Yongtao Liu $\left(\mathbb{D},{ }^{1}\right.$ Jie Qiao $\left(\mathbb{D},{ }^{1}\right.$ Tianyuan Han, ${ }^{1}$ Longhui $\mathrm{Li}^{2}$ and Ting $\mathrm{Xu}$ (D) ${ }^{3}$ \\ ${ }^{1}$ School of Automobile, Chang'an University, Xi'an 710064, China \\ ${ }^{2}$ Automotive Data of China Co., Ltd., Tianjin 300300, China \\ ${ }^{3}$ College of Transportation Engineering, Chang'an University, Xi'an 710064, China \\ Correspondence should be addressed to Jie Qiao; 2016022001@chd.edu.cn and Ting Xu; 850573553@qq.com
}

Received 2 June 2020; Revised 14 August 2020; Accepted 16 November 2020; Published 23 December 2020

Academic Editor: Francesco Galante

Copyright (C) 2020 Yongtao Liu et al. This is an open access article distributed under the Creative Commons Attribution License, which permits unrestricted use, distribution, and reproduction in any medium, provided the original work is properly cited.

Long tunnels often collapse during the construction period. To ensure personnel safety, the geological characteristics must be predicted before tunnel face excavation. In this study, the ground-penetrating radar (GPR) technique is introduced to obtain information regarding the tunnel excavation face at a certain interval. The amplitude of the radar echo signal is expressed as a function of the position and travel time. A B-scan strategy is selected for the GPR to obtain tunnel information. A frequencydomain $(w-k)$ focusing algorithm, namely, a synthetic aperture radar focusing algorithm, is applied to focus scattered radar signals to obtain focused images. A low-pass filter is designed to remove noises from the original signals. The contours of target objects are extracted from the background information using the edge detection technique. Space coordinate values of the objects are converted to polar coordinates using the Hough transform algorithm for 3D modeling. Visual C++ and AutoCAD are combined to develop a 3D CAD model to help managers in controlling the construction process. The system creates 3D visualization model images and evaluates the geological characteristics of the tunnel excavation faces. The Taigu Tunnel located in the Shanxi Province of China is taken as a case study. A procedure for the geological analysis of this tunnel is introduced in detail, and a 3D image model is built. The results show that the 3D model can help predict rock compositions and locate potential hazards. Moreover, it has better accuracy than conventional models and can be applied to similar transportation construction projects.

\section{Introduction}

China has the largest and widest network of traffic tunnels in the world. By the end of 2018, the number of road tunnels alone is expected to reach $17,738 / 17.236$ million linear meters, of which 1,058 will be extralong tunnels per 4.7065 million linear meters and 4,315 will be long tunnels per 7.421 million linear meters $[1,2]$. The construction of such tunnels is associated with significant construction difficulties and risks, including high geostresses and many geological problems such as high geotemperature, high hydraulic pressure, special adverse strata, high-pressure water-rich karsts, large deformation of soft rock, and large deformation of the Archaean metamorphic hard rock [3, 4]. Tunnel collapse, roof caving, water inrush, and/or other accidents increase the construction difficulty and construction cost; the safety of personnel is another major concern [5-7]. Visualizing a geological structure through $3 \mathrm{D}$ modeling is considered an effective method for predicting rock composition and determining potential hazards.

Geological structure prediction is an essential routine work in the excavation of tunnels, providing important prior information that can help ensure a safe, economical, and efficient tunneling [8-11]. Several methodologies have been developed for the geological prediction of tunnel rocks, such as surface investigation, geological analysis, geophysical prospecting, and rock mapping. These efficient and unbiased methods for hazardous inspection can be grouped into two categories: destructive and nondestructive methods [12]. As early as the 1950s, geological structures were being analyzed by directly drilling the tunnel faces [13]. This destructive method can help access the rock samples ahead of the 
tunnels and the surrounding rocks. However, it is timeconsuming and costly. Nondestructive methods for geological prediction during tunnel construction saw their emergence in Europe in the 1980s and America and China in the 1990s. Unlike destructive methods, nondestructive methods are designed to provide information about the geological properties without deteriorating the material microstructure, making them suitable for the geological forecasting of tunnel faces. Nowadays, geological prospecting has been developed, ranging from destructive methods such as drilling and pilot tunnel excavation to nondestructive methods such as mechanical, electromagnetic, and electrical methods [14]. Each approach has its advantages and disadvantages. Zhang et al. recommended the seismic reflection method, ground-penetrating radar (GPR), infrared water detection, a transient electromagnetic method, and advanced probe borehole drilling to predict the location, size, and distribution of karst features ahead of tunnel faces [15]. Yuan et al. conducted a comprehensive investigation through geophysical exploration, deep hole drilling, and ground stress testing to determine the geological and hydrogeological conditions of a tunnel [16]. The mechanical methods include negative apparent velocity, seismic, ultrasonic, and acoustic emission methods. Wang et al. detected hidden structures in a tunnel surrounded by rocks using the pulse reflection method [17]. Jiang et al. applied a joint seismic travel time and waveform inversion method to image a buried tunnel with concrete walls and a void space inside [18]. Zhang et al. proposed an improved shallow soil-rock interface seismic reflection method for a geotechnical field investigation [19]. However, this seismic method is not ideal as it is difficult to accurately determine the seismic velocity of the rocks around the tunnel walls. These methods may yield inaccurate geological inferences, which will hamper the classification of the wall-rock characteristics. Considering the drawbacks of the seismic method for hydrogeological surveys, Liu et al. used the seismic and resistivity methods to predict tunnel collapse and water inrush due to fracture zones and water-bearing structures [20]. Pazzi et al. conducted a comprehensive geophysical survey using resistivity tomography, microgravity method, and single-station seismic noise measurement to explore the cause of a massive sinkhole called Tiankeng [21]. Computer techniques have been successfully implemented in the construction industry for decision-making and monitoring.

Electrical methods include electromagnetic (EM) and electrical resistivity (ER) investigations. The inspection requires special measuring devices and complex investigations of the information contained in the measured signals. The receiver records the signal strength at certain points on the near-surface to depths greater than $300 \mathrm{ft}$. The ratio of the voltage to the current is the resistance. When the measurement is made over a homogeneous surface, the apparent resistivity is equal to the true resistivity of the ground. Resistivity imaging is a technique used to detect and characterize water bodies in open fractures or faults based on the resistivity contrast between the water bodies and surrounding materials [22]. Ismail established a 3D filtered tunnel model of the weathering grade, quality grade, and heat capacity of rock mass based on surface survey data and electrical resistivity tomography technology [23]. Recently, the GPR, which is an electromagnetic method, has been considered the most useful technique to search for metals and minerals at relatively shallow depths (approximately 15-30 m). A GPR comprises a radar antenna that transmits and receives wide-frequency electromagnetic energy impulses at frequencies between $25 \mathrm{MHz}$ and $1 \mathrm{GHz}$. The different dielectric constants of the medium will produce electromagnetic wave reflection and the amplitude of the received electromagnetic waves. The frequency characteristics, subsurface structure, and stratigraphic characteristics can be identified using geophysical technology with high observational accuracy. This method has been successfully applied to map subsurface geological structures and groundwater contaminants. In 1988, Daniels et al. published a tutorial paper entitled "Introduction to subsurface radar," giving a good overview of the GPR technology. The GPR is widely used in traffic infrastructure and tunnel detection because it can accurately and continuously detect the contours of road surfaces [24]. Li et al. used GPR to measure tunnel lining thickness [25]. Li et al. comprehensively applied GPR and Geo-3d technology to survey the geometric characteristics of karst caves and their spatial relationship with tunnels [26]. Alani et al. and Zhang et al. studied the relationship between different detection frequencies of GPR and imaging resolution and detection depth [27, 28]. Wei et al. found that integrating the data obtained by multiple GPRs and seismic sensors can help recover the 3D position of possible events in front of the mining face [29].

This study is focused on controlling geological hazards in advance during the evacuation period using nondestructive methods. The GPR technology is used to capture information regarding the rock structures of excavation faces. To extract the features of the tunnel faces, radar reflection is transformed into digital images using synthetic aperture radar (SAR) algorithm. The rock conditions can be easily interpreted and conceived in a $3 \mathrm{D}$ environment. Typically, two-dimensional maps are applied to show the locations and directions of the weak planes, and the excavation face conditions are determined by manual visual investigation for most tunnels. It is a costly and time-consuming process and even extremely dangerous. In this regard, Zhao et al. conducted a $3 \mathrm{D}$ simulation on the complex failure evolution of a brittle fault zone and quantitatively evaluated the potential failure mode [30]. Liu et al. established an overall high-precision $3 \mathrm{D}$ model by comprehensively considering the topography, folds, and major faults and revealed the zoning phenomenon of the in situ stress along the line [31]. Vanneschi et al. established a 3D model of a geological structure by combining 3D laser scanning technology and photographic image technology [32]. Xiong et al. proposed a $3 \mathrm{D}$ multiscale geological modeling method for the risk assessment in tunnel engineering by coupling the surrounding rock geological body model and a tunnel model [33]. With the development of information management systems, the use of 3D CAD models has become increasingly common in construction projects. A 3D CAD model can help visualize a project in $3 \mathrm{D}$, and tunnel-related characteristics can be 
recognized using a computer system, thus enabling the efficient control of the construction process. Tonini et al. proposed a geological modeling method based on field survey data and reconstruction of a geological section perpendicular to a geological structure (fold) axis (geological section) using a CAD software system [34]. Thum proposed a method based on a digital geographic information system (GIS) to conduct a geological survey and realized the automation of geological and geomechanical mapping in the process of tunnel excavation [35].

The rest of this paper is organized as follows. First, the tunnel face detection technique GPR is briefly introduced. Subsequently, the B-scan strategy for tunnels is reviewed. In the third part, we discuss the SAR algorithm and Hough transform for 3D image model construction. Computerized software for 3D tunnel modeling is illustrated in the fourth part. In the discussion part, the Taigu Tunnel project undertaken by China is taken as a case study to demonstrate the efficiency of the software. Finally, the conclusions are summarized.

\section{GPR Theory}

The precision and reliability of conventional detection methods were improved in this study. Since tunnel rocks have varied shape, size, and depth and are filled with a variety of materials, the selection of the geophysical technique for the investigation depends on many variables.

Underground excavation is associated with many problems related to safety. Since complete records of tunnel construction are unavailable, common radar images are a valuable source to interpret geological information [36]. In this study, the GPR was selected to inspect the different layers of a tunnel; this method uses radio waves to probe the natural geological material. In a typical GPR measurement process, a transmitter and a receiver are deployed in a fixed geometry and are moved over the surface to detect any reflections from subsurface features, as shown in Figure 1.

The GPR antenna moves horizontally across the surface of the ground. The transmitting antenna radiates short high-frequency electromagnetic pulses into the ground, where they are refracted, diffracted, and reflected as they encounter variations in the dielectric permittivity and electric conductivity. The most common operation mode of the GPR for detecting tunnel face geological structures is the reflection mode, whereby the traces of returned waves are collected either continuously or in stations along a line, thus creating a time cross section or a profile image of the subsurface. The velocity of the waves varies with the physical and chemical properties of the material through which they travel.

Signals transmit electromagnetic pulses from the surface antennas into the ground. The time elapsed between sending the pulses and receiving them back at the surface is measured [37]. When the travel times of the energy pulses are measured and their velocity through the ground is known, the distance can be accurately determined. The radar travel time is calculated using the following equation:

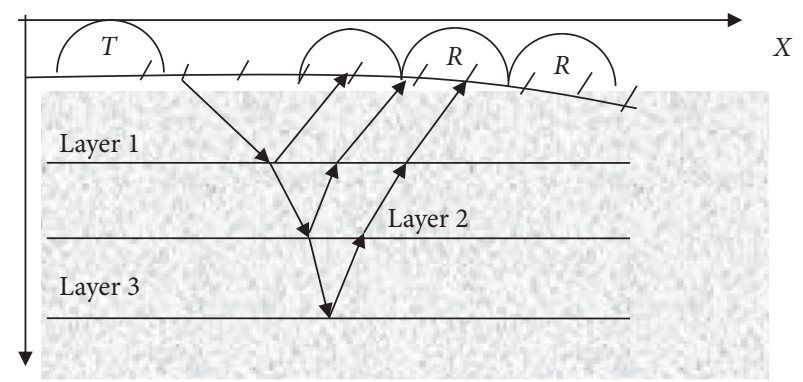

FIGURE 1: Flowchart of a typical ground-penetrating radar system.

$$
t=\frac{\sqrt{4 z^{2}+x^{2}}}{v} \approx \frac{2 z}{v}
$$

where $z$ is the distance to the reflectors, in $m$; $t$ is the two-way travel time, in ns; $x$ is the distance between the antenna and receiver; and $v$ is the velocity of the electromagnetic waves in the medium, which is calculated using the following equation:

$$
v=\frac{c}{\sqrt{\varepsilon_{r} \mu_{r}}} \approx \frac{c}{\sqrt{\varepsilon_{r}}},
$$

where $c$ is the propagation velocity of the electromagnetic waves in vacuum $(0.29979 \mathrm{~m} / \mathrm{ns}) ; \varepsilon_{r}$ is the relative permittivity of the subsurface material; and $\mu_{r}$ is the relative permeability of the magnetic medium.

The electromagnetic wave velocity is equal to the product of the wavelength $\lambda$ and the frequency $f$ as expressed in the following equation:

$$
v=\lambda f .
$$

The transmission frequency $f$ in a GPR survey ranges from 25 to $100 \mathrm{MHz}$.

When the electromagnetic wave propagation changes due to the relative permittivity under geological conditions, the electromagnetic waves will produce reflection and transmission, and their energy allocation is closely related to the abnormal changes in the electromagnetic wave reflection coefficient. The reflection coefficient is calculated using the following equation:

$$
y=\frac{\left(\varepsilon_{1}^{1 / 2}-\varepsilon_{2}^{1 / 2}\right)}{\left(\varepsilon_{1}^{1 / 2}+\varepsilon_{2}^{1 / 2}\right)},
$$

where $y$ is the reflection coefficient of the electromagnetic waves at the interface and $\varepsilon_{1}$ and $\varepsilon_{2}$ are the relative dielectric constants of the first and second layers, respectively.

\section{GPR Scanning Strategy}

The radar feedback signal is processed to form an amplitude image of the tunnel faces ahead via different scanning strategies. The algorithms can be divided into A-scan, $\mathrm{B}$-scan, and C-scan depending on the scanning geometries and directions. A-scan screening involves recording two parameters: amplitude and pulse transit time. The transit time of the radar echoes is used to fix the distance of the 
objects and tells the observer of certain interfaces along a line by one dimension.

However, 1D image information is not useful for tunnel face evaluation. Many researchers prefer at least a 2D image than a simple one-dimensional image because it can be used to take an image of a cross section through the object.

Hence, we employed the B-scan GPR algorithm to show weak planes. The scattered data were recorded from several spatial positions on a straight scan by moving antennas on the tunnel surfaces and looking downward. As shown in Figure 2, the antenna moves along the $x$ direction, and a series of pulse-echo measurements are acquired for all $(x, y)$ positions over a plane, where $z$ is the space depth.

The individual reflected waves received are digitized into a reflection trace, and when many traces are stacked next to each other, a $2 \mathrm{D}$ vertical profile can be produced along the transect. The set of A-scans is gathered to form a 2D dataset $b(x, t)$, called the B-Scan, as shown in Figure 3.

In the B-scan, the vertical axis represents the screening time and the horizontal axis represents the radar scan traces [38]. During this process, a transmission and reception phenomenon occurs at each observation position, and then, the recorded data are combined to produce a $2 \mathrm{D}$ space-time GPR image.

\section{GPR Focusing Algorithms}

Because of the beam width of the transmitting and receiving antennas, the reflections on a structure spread over a broad region in the recorded data. Many focusing techniques have been developed to correct defocusing images. The SAR algorithm is applied to original GPR B-scan images to obtain true information about the location, shape, and size of targets. Furthermore, the SAR focusing algorithm is efficient and does not introduce additional losses to the digital signal acquisition system. Since basic B-scan signals in the time domain cannot handle waves of varying speed, the frequency-domain $w$ - $k$ method based on the SAR is introduced in this study.

In this study, a 2D Fourier transform is applied to interpolate the signal in the Fourier space. As shown in Figure 3, the antenna moves along the $X$ axis. The magnitude and phase of the EM scatterings are recorded at each discrete point. Suppose the target point $P$ is located at ( $x i$ and $z i$ ), the recorded data $E s(x, \omega)$ in the frequency domain can be written as follows [39-41]:

$$
E s(x, \omega)=\sum_{i=1}^{p} \rho_{i} e^{-j 2(\omega / v) \sqrt{z_{i}^{2}+\left(x-x_{i}\right)^{2}}},
$$

where $\omega=2 \pi f$ is taken for convenience of nomenclature, $\rho$ is the complex amplitude of the scattered electric field of the point target, and $v$ is the velocity of the electromagnetic waves in the medium, as expressed in equation (2).

By applying 1D Fourier transform to $E s(x, \omega)$ from space $x$ to spatial frequency $k_{x}$, we can obtain the $w$ - $k$ domain representation of the scattered data using the following equation:

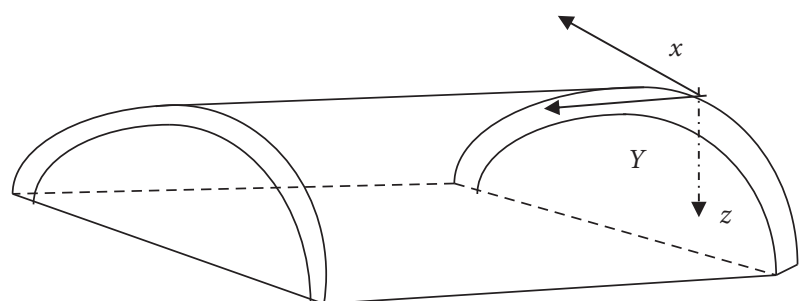

Figure 2: Radar B-scanning scheme for tunnels.

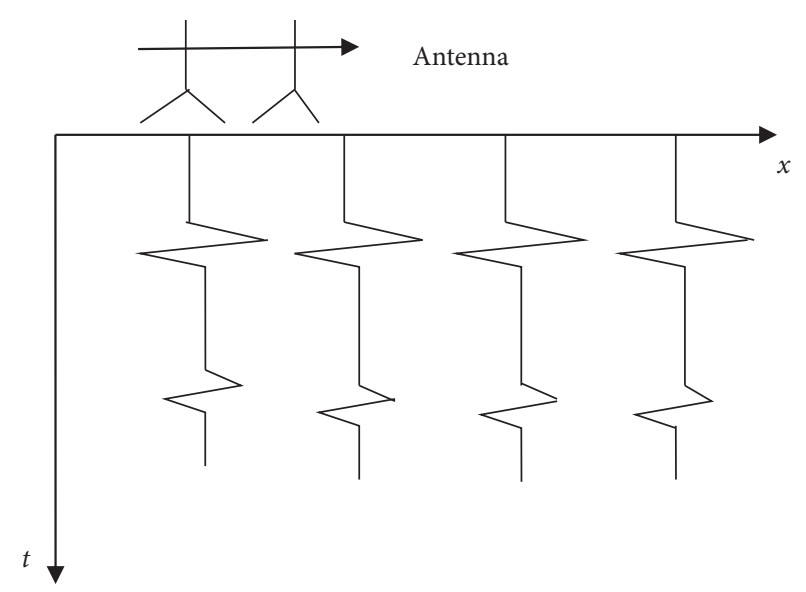

Figure 3: B-scan strategy.

$$
E s\left(k_{x}, \omega\right)=\sum_{i=1}^{p} \rho_{i} \int_{-\infty}^{\infty} e^{-j 2(\omega / v) \sqrt{z_{i}^{2}+\left(x-x_{i}\right)^{2}}} e^{j k_{x} x} \mathrm{~d} x .
$$

Two-dimensional $E s^{\prime}\left(k_{x}, k_{z}\right)$ data can be obtained by interpolating $E s\left(k_{x}, \omega\right)$ onto a $\left(k_{x}, k_{z}\right)$ mesh. Thereafter, the final focused GPR image can be obtained by transforming the data from the $\left(k_{x}, k_{z}\right)$ domain to the $(x, z)$ domain via the following equation:

$$
e(x, z)=\frac{1}{(2 \pi)^{2}} \int_{-\infty}^{+\infty} \int_{-\infty}^{\infty} E_{s}^{\prime}\left(k_{x}, k_{z}\right)^{e^{j\left(k_{x} x+k_{z} z\right) d k_{x} \mathrm{~d} k_{z}}},
$$

where $e(x, z)$ is the focused GPR image in the spatial-spatial domain after the $w$ - $k$ domain SAR algorithm.

\section{Image Model Reconstruction}

5.1. Signal Processing. Synthetic aperture radar images contain speckle noise that degrades the image quality, and the noise can significantly impact the performance of the radar in judging the target [42]. Although weak planes can be detected and located by the SAR algorithm, the exact material depth in the layers should be determined using the signal process techniques. In this study, a low-pass filter was used to detect images and suppress noise. All the noise signals are considered high-frequency noise and have a smoothing effect for images.

It is better to perform contour detection automatically after the low-pass filter process. Edges define the boundaries between the regions in an image, which helps with segmentation, and the object contour position of each geological layer is calculated using an edge detection recognition 
algorithm. To evaluate the number of fringes quickly and exactly, an extreme point tracking method is proposed in this study. A $3 \times 3$ window is applied to the images with a certain threshold. The threshold is recalculated as the mean of nine intensity values of the pixels in the window. If a pixel has an intensity value greater than this threshold, it is set to 1 ; otherwise, it is set to 0 . The original image is converted to a binary pattern with a $3 \times 3$ window. Sixteen possible edgelike patterns arise in $3 \times 3$ windows, as shown in Figure 4 .

If the binary window obtained above matches any of the sixteen masks, the center pixel of the window is set to be an edge pixel. The previous steps are then repeated for each pixel in the image as the center pixel of the window, and all edges can be determined. To remove false edges due to noise, the variance for each $3 \times 3$ window is calculated and then compared with a global threshold based on the level of noise in the image. If the value is greater than the threshold, it is retained as an edge. If it is not greater than the threshold, it is removed.

Figure 5 shows the schematic diagram of the algorithm.

5.2. Three-Dimensional CAD Model. With the development of computer technology, 3D CAD models are being applied to visualize processes during the construction phase, instead of $2 \mathrm{D}$ images. Three-dimensional tunnel modeling not only involves geometrical data reconstruction but also texture extraction.

The Hough transform technique is used to calculate the tunnel position parameters in space, which can be used to isolate the features of a particular shape within an image. To simplify the $3 \mathrm{D}$ visualizing process, all the geological layers are considered regular shapes. Hence, the classical Hough transform is applied to $3 \mathrm{D}$ tunnel modeling since it is concerned with the identification of characteristics in the image.

We take the simplest linear transform as an example; a straight line can be expressed by $y=a x+b$ in image space and can be graphically plotted for each pair of image points $(x, y)$. In the Hough transform, a straight line is considered a function of the slope $a$ and the intercept parameter $b$. Hence, the straight line can be represented as a point $(a, b)$. For computational reasons, it is better to use a different pair of parameters, namely, $r$ and $\theta$, for the lines in the Hough transform, as shown in Figure 6.

The parameter $r$ represents the distance between the line and the origin, and $\theta$ is the angle of the vector from the origin to this closest point. Using this parameterization, we can express the equation of a line as follows:

$$
y=\left(-\frac{\cos \theta}{\sin \theta}\right) x+\left(\frac{r}{\sin \theta}\right),
$$

where $r \in R, \theta \in[0,2 \pi], r \geq 0$, and for straight lines in two dimensions, we can rewrite the equation as $r(\theta)=x_{0} \cos \theta+y_{0} \sin \theta$. Hence, it is possible to associate with each line of a pair.
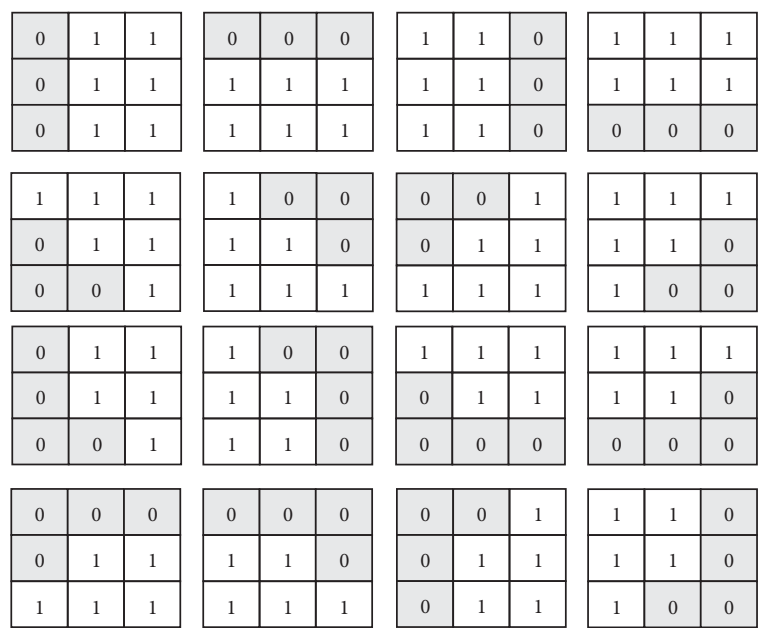

FIgURe 4: 16 possible binary patterns of images.

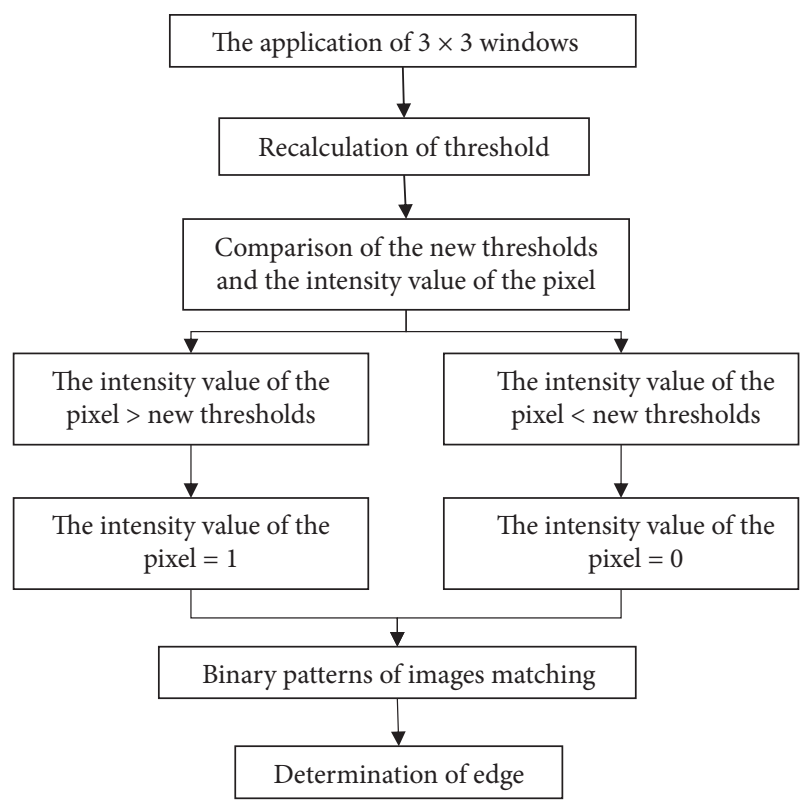

FIgURE 5: The schematic diagram of the algorithm.

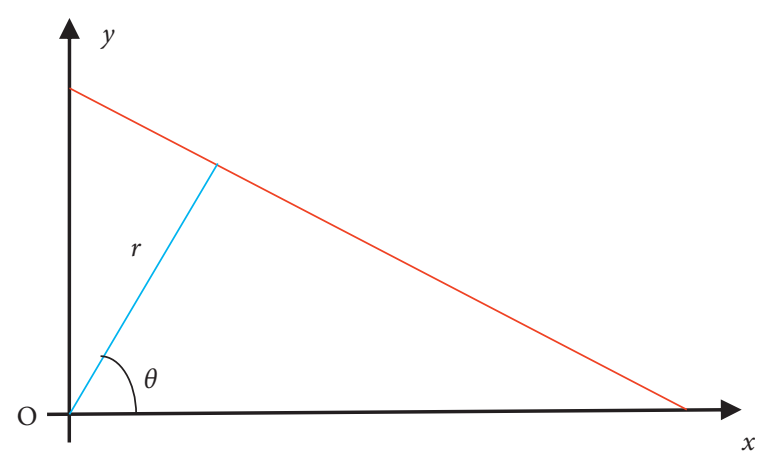

FIgURE 6: Lines in polar coordinates. 


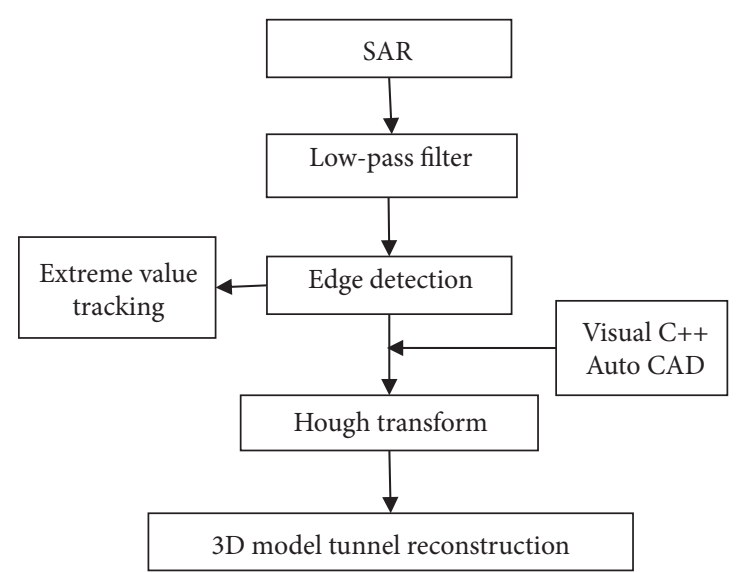

FIgURE 7: Procedure for tunnel reconstruction.

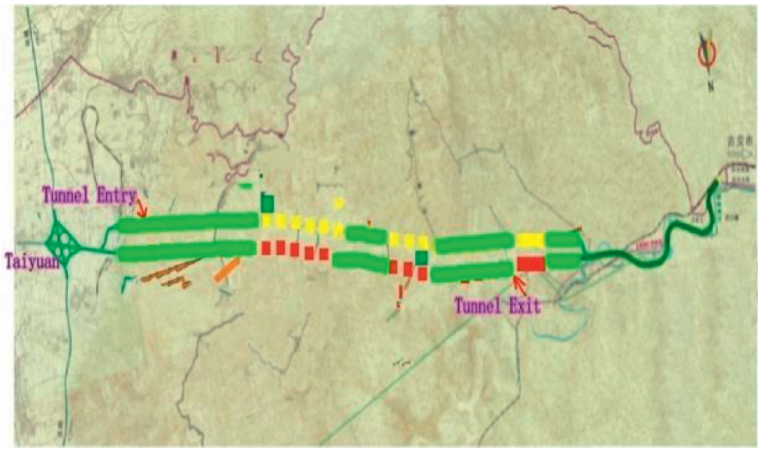

(a)

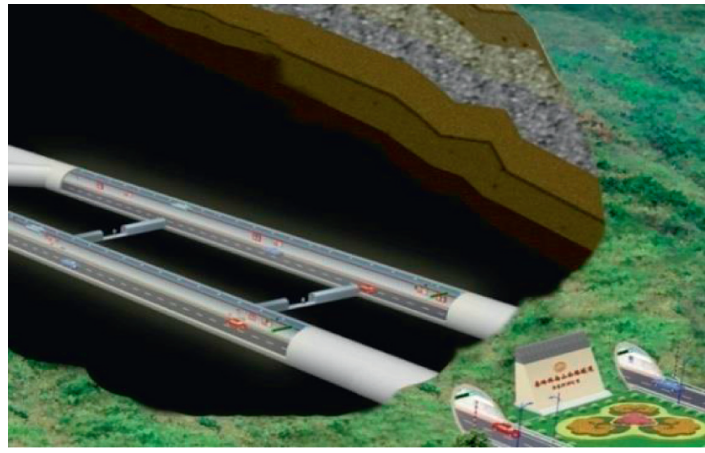

(b)

Figure 8: Taigu Tunnel.

To obtain a 3D model of a tunnel, the Hough transform concept is extended to 3D space. Suppose any plane in the $X$ $Y-Z$ space corresponds to a $\rho-\theta-\varphi$ space and considering a particular point $A\left(x_{a}, y_{a}, z_{a}\right)$ in the $X-Y-Z$ space, planes passing through this point can be expressed as in the following equation:

$$
\rho=x_{a} \sin \theta \cos \phi+y_{a} \sin \theta \sin \phi+z_{a} \cos \theta,
$$

where $(\rho, \theta, \varphi)$ is a vector from the origin to the nearest point on the plane. In the study, all the contour points of the SAR images in the $\rho-\theta-\varphi$ space were converted to the spatial domain using the Hough transform technique.

AutoCAD and Visual C++ software package were used to model the 3D tunnel image. Tunnel shaped data are obtained by on-site measurement at each cross-section along the $X$ axis. The appropriate values of the length are determined based on the evacuation process. Three-dimensional models are generated from the cross-section of the tunnels obtained using the software. The software maps the entire model of the images and extracts the tunnel texture.

Figure 7 shows the procedure for establishing a 3D tunnel model.

\section{Discussion}

This paper discusses a three-dimensional modeling system of tunnel construction, which can establish the three-dimensional model of tunnel and then ensure the safety of tunnel construction. The following case study demonstrates the benefits of the 3D modeling system for tunnel construction projects. The 3D modeling system was developed using Autodesk AutoCAD 2010 and Visual C++ tool. The system is used to analyze the rock geological layers along the drilling direction and visualize the drilling process. Potential hazards are identified in advance to ensure safety during construction.

The project is the construction of the Shanxi Long Tunnel, a section of the Taigu Tunnel, which is the secondlongest tunnel in China. It is located in the east side of the Lu-Liang mountainous area in Shanxi Province and passes through coal seams, goaves, and other adverse geological environments, as shown in Figure 8.

The tunnel is characterized by a split pattern design. The left way is $13654 \mathrm{~m}$ long, and the right way is $13570 \mathrm{~m}$. The construction sections start from $\mathrm{K} 0+900$ and end at $\mathrm{K} 7+550 \mathrm{~m}$. The tunnel altitude is between 887.92 and $1385.58 \mathrm{~m}$ 


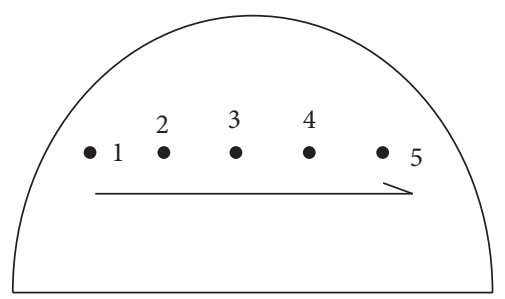

(a)

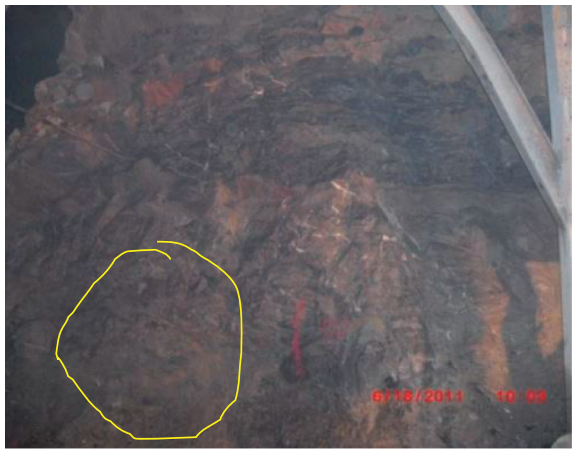

(c)

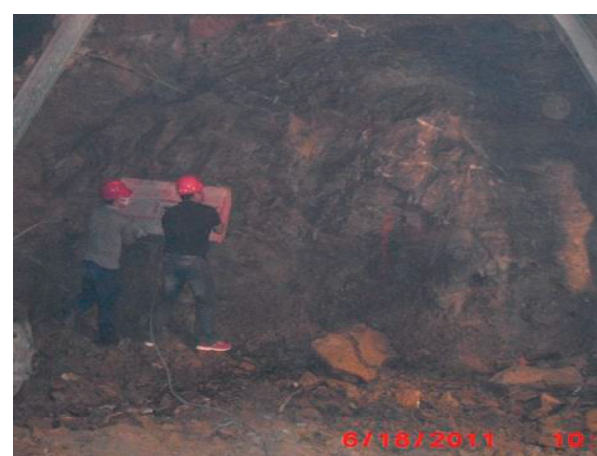

(b)

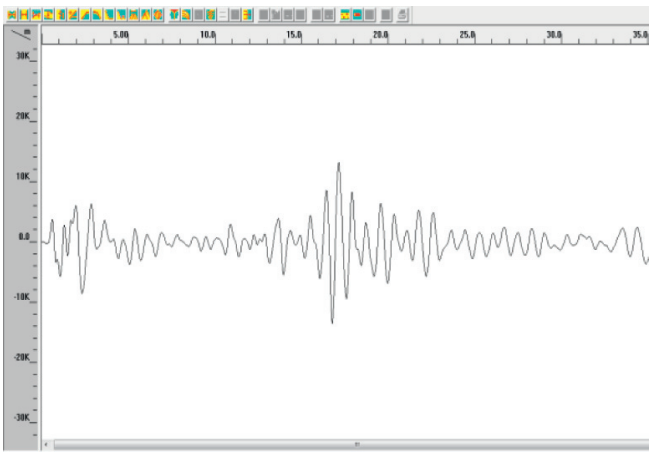

(d)

FIgURE 9: Antenna and markings. (a) Layout of testing points. (b) Radar antenna. (c) Radar marking on the wall. (d) Original radar signals.

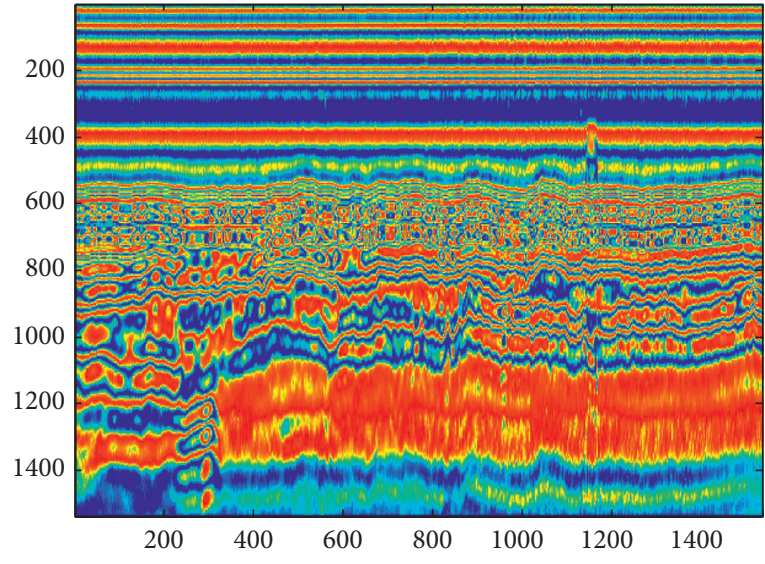

(a)

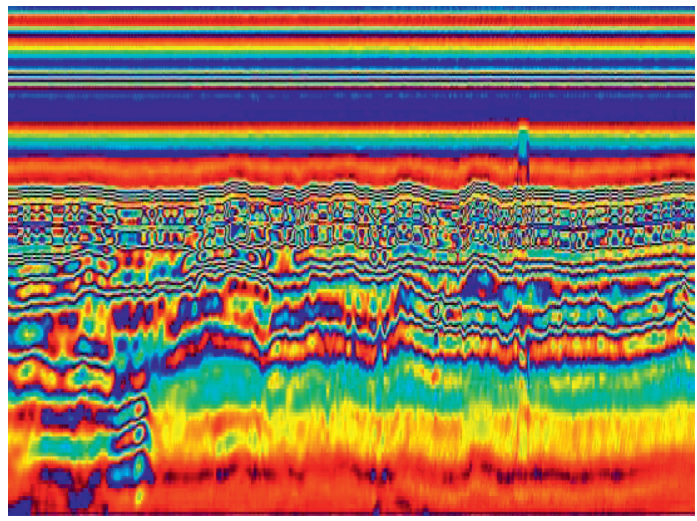

(b)

Figure 10: Continued. 


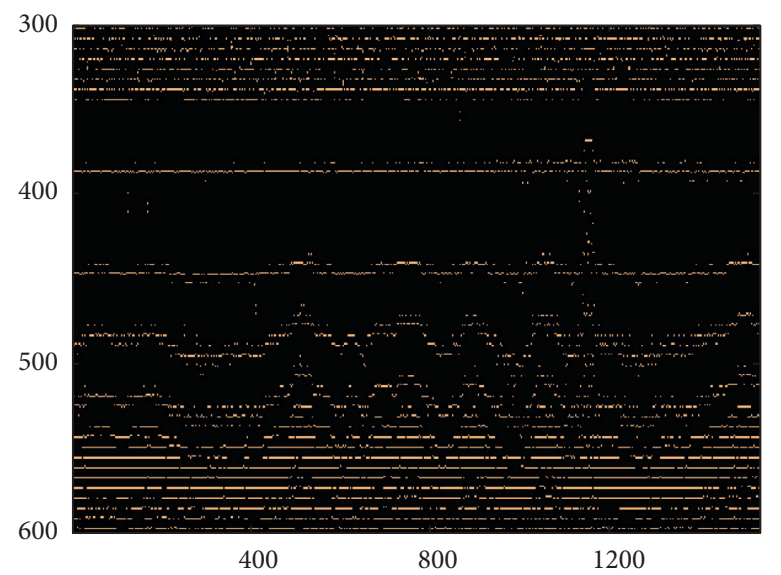

(c)

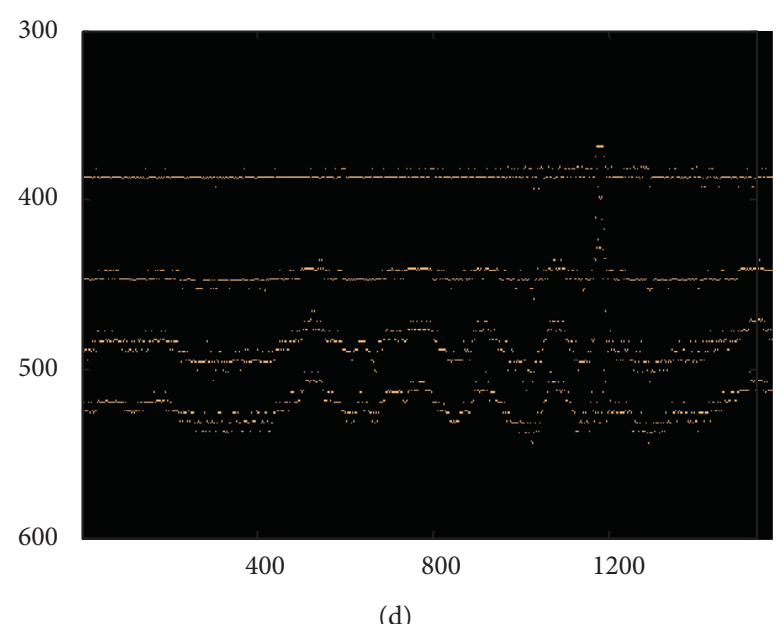

(d)

Figure 10: Image analysis: (a) focusing images, (b) image after noise reduction, (c) edge extraction, and (d) noise reduction.

The access of No. 1 shaft of the tunnel is at YK $4+488$ at the right way and crosses the main hole at YK5 +477 with a $25^{\circ}$ slope. The angle between the mainline and the horizontal projection is $5.9^{\circ}$. The project was to be completed in December 2014. Owing to the absence of a reference coordinate system for the long tunnel, a hand measuring wheel was made to run along the tunnel to paint marks on the wall at $15 \mathrm{~m}$ intervals to increase the positioning accuracy.

Conventional geological forecasting involves tunnel rock monitoring, hydrogeologic monitoring, geological mapping, and rock category identification. In this study, a SIR-3000 series GPR manufactured in the US was selected to detect the geological layer, and its frequency is $100 \mathrm{MHz}$. A continuous reading of the radar detection signal was completed manually at the marked points. It took approximately $15-20 \mathrm{~min}$ to capture the images for one round. The radar images were processed in the control center at the entrance of the tunnel. Figure 9(a) shows the cross-sectional view of five testing points. Figure 9(b) shows the radar antenna. Figure 9(c) shows the marking on the wall for the testing points. Figure $9(\mathrm{~d})$ shows the recorded original signals.

The two-dimensional datasets obtained using the GPR in the time-domain were collected, including the original echo signals from the tunnel surfaces. However, it was difficult to extract the target from the background signals shown in Figure 10. The SAR was applied to focus the original beams. The radar speed and echo depth were analyzed simultaneously. Figure 10(a) shows the SAR image. The straight lines indicate the radar beams. A low-pass filter was used for noise reduction. Figure 10(b) shows the image after denoising. An edge detection technique was used to determine the contours of each geological layer. Figure 10(c) shows the edge extraction process. It can be seen from the Figure $10(c)$ that there are many noise signals, which need to be processed. The lines in the images indicate noise generated due to the coupling of the transmitting and receiving antenna, and an adaptive method is used to eliminate this noise, as shown in Figure 10(d).

OPGL package and $\mathrm{C}++$ combined with AutoCAD were used to develop the computer software to visualize the

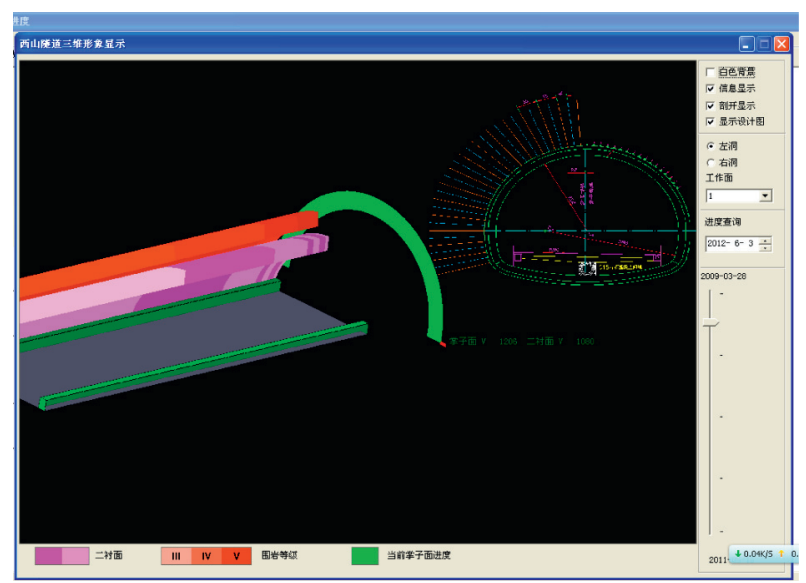

FIgURE 11: Three-dimensional tunnel model software.

process of tunnel excavation. The design data were stored in the database in advance and compared with the process in practice. The points in the $2 \mathrm{D}$ spaces were projected into a 3D space by Hough transform. Figure 10 illustrates the historical excavation process and construction process. The left part is the 3D tunnel model of the geological structure. The red color indicates the rock layers, and the purple color indicates the tunnel lining. The green color indicates the excavation face. The cross-sectional view is displayed in the right part of the software interface. The right and left holes can be displayed based on user requirement, as shown in Figure 11.

It can be seen from Figure 11 that this method can more accurately estimate the geological characteristics of the tunnel, which is beneficial to ensure safety during the actual construction of a long tunnel.

\section{Conclusions}

The hazard risk during the construction period of a tunnel should be identified in advance. In this study, the GPR detection technique was applied to obtain the geotechnical 
features of a tunnel. The original radar signals were collected at five points. After the analysis, the following conclusions were obtained:

(1) The B-scan strategy was used to obtain 2D images from the cross-sectional view when the antenna moves along the $x$ direction. The SAR algorithm was applied to the original signals to focus all the beams at certain points.

(2) Since the basic B-scan signals in the time domain cannot handle waves of varying speed, a 2D Fourier transform was applied to interpolate the signal into the Fourier space based on the SAR algorithm.

(3) The signal processing consists of two phases: lowpass filtering and edge detection. In this study, a low-pass filter was used to detect images and suppress the noise. All the noise signals were considered high-frequency components, with a smoothing effect for images. The contour position of each geological layer was calculated using edge detection recognition.

(4) A 3D modeling software was developed using Visual $\mathrm{C}++$ for a better analysis of the tunnel construction process. The $3 \mathrm{D}$ model produces animations that serve as accurate representations to predict the construction process, thus facilitating the control of the construction process and identification of geological risks.

(5) The Taigu Tunnel project was taken as an example to demonstrate the efficiency of the developed software. Different rock layers could be visualized using the 3D model, including the tunnel cross-sectional view. The 3D computerized model can also be applied to other construction projects.

\section{Data Availability}

The data used to support the findings of this study are available from the corresponding author upon request.

\section{Conflicts of Interest}

The authors declare that there are no conflicts of interest regarding the publication of this paper.

\section{Acknowledgments}

The authors would like to thank Chang'an University for providing equipment. Also, they specially thank the graduate students who helped them in data collection. The project was supported by the National Science of Foundation of China (Grant nos. U1664264 and 51878066), the Humanities and Social Sciences Youth Fund of Ministry of Education (18YJCZH110), and Fundamental Research Funds for the Central Universities in China (300102220111, 300102120111, 300102229201, and 300102229668).

\section{References}

[1] Ministry of Transport of the People's Republic of China, Interpretation of the Action Plan for Improving the Quality and Upgrading of Highway Tunnels, Ministry of Transport of the People's Republic of China, Beijing, China, 2019, http://www. mot.gov.cn/zxft2019/tizhishj/index.html.

[2] J. X. Yan, "Achievements and challenges of tunneling technology in China over past 40 years," Tunnel Construction, vol. 39, no. 4, pp. 537-544, 2019.

[3] H. Zhu, J. Yan, and W. Liang, "Challenges and development prospects of ultra-long and ultra-deep mountain tunnels," Engineering, vol. 5, no. 3, pp. 384-392, 2019.

[4] Z. N. He, "Analysis of geological problems of railway tunnels: case study of typical tunnels," Tunnel Construction, vol. 36, no. 6, pp. 636-665, 2016.

[5] Y. Shang, W. Pang, K. Wang et al., "Deformation damage of shallow tunnels and caveinduced ground collapse in archeozoic gneissic granites-the Jining tunnel as case example," Journal of Engineering Geology, vol. 26, no. 6, pp. 1574-1584, 2018.

[6] W. X. Wang, Z. H. Zhang, Y. Zhou, S. P. Wu, and Y. X. Ran, "Construction technology of shallow buried gravel soil section of yanchong highway Xinglinbao tunnel under S241 Province road," Highway, vol. 65, no. 3, pp. 349-352, 2020.

[7] J. Shao, "Research on the large water Inrush mechanism and water pressure of Feixianguan tunnel in red bed area," Highway, vol. 65, no. 2, pp. 294-300, 2020.

[8] W. Zhao, "Analysis of main geological problems in altun region and countermeasures of railway alignment selection," Railway Engineering, vol. 59, no. 3, pp. 97-103, 2019.

[9] X. Cao, "Schematic study of central and Southern Shanxi railway passing through Lvliang Mountains," Railway Standard Design, vol. 58, no. 2, pp. 8-12, 2014.

[10] Y. H. Zhao, "Research of integrated route scheme for Xi'anpingliang railway passing through yongshouliang mountain region," Railway Standard Design, vol. 58, no. 2, pp. 8-12, 2014.

[11] G. W. Li, Y. B. Du, L. W. Jiang et al., "Research on the engineering geology condition and railway routes comparison along the Mt.gaoligong section, dali-ruili railway," Journal of Geomechanics, vol. 21, no. 1, pp. 73-86, 2015.

[12] H. Madani, Tunneling Sed, Tehran Polytechnic University Press, Tehren, Iran, 1998.

[13] P. J. Tarkoy and J. E. Byram, The Advantages of Tunnel Boring: A Qualitative Comparison of D\&B and TBM Excavation, Hong Kong Engineer, Hong Kong, China, 1991.

[14] S. C. Li, B. Liu, X. J. Xu et al., "An overview of ahead geological prospecting in tunneling," Tunnelling and Underground Space Technology, vol. 63, pp. 69-94, 2017.

[15] K. Zhang, D. D. Tannant, W. Zheng, S. Chen, and X. Tan, "Prediction of karst for tunnelling using fuzzy assessment combined with geological investigations," Tunnelling and Underground Space Technology, vol. 80, pp. 64-77, 2018.

[16] Y. T. Yuan, "Application of comprehensive survey technology for super long tunnel in altun mountain," Journal of Shandong Agricultural University(Natural Science Edition), vol. 49, no. 4, pp. 606-610, 2018.

[17] J. C. Wang, C. Y. Wang, Z. Q. Han, Y. Y. Jiao, and J. P. Zou, "Study of hidden structure detection for tunnel surrounding rock with pulse reflection method," Measurement, vol. 159, Article ID 107791, 2020. 
[18] W. B. Jiang, C. A. Zelt, and J. Zhang, "Detecting an underground tunnel by applying joint traveltime and waveform inversion," Journal of Applied Geophysics, vol. 174, Article ID 103957, 2020.

[19] Y. H. Zhang, Y. Y. E. Li, and T. Ku, "A modified seismic reflection approach for engineering geology investigation in fractured rock zones," Engineering Geology, vol. 270, Article ID 105592, 2020.

[20] B. Liu, Q. Guo, Z. Y. Liu et al., "Comprehensive ahead prospecting for hard rock TBM tunneling in complex limestone geology: a case study in Jilin, China," Tunnelling and Underground Space Technology, vol. 93, Article ID 103045, 2019.

[21] V. Pazzi, M. Di Filippo, M. Di Nezza et al., "Integrated geophysical survey in a sinkhole-prone area: microgravity, electrical resistivity tomographies, and seismic noise measurements to delimit its extension," Engineering Geology, vol. 243, pp. 282-293, 2018.

[22] S. Li, S. Xu, L. Nie et al., "Assessment of electrical resistivity imaging for pre-tunneling geological characterization - a case study of the Qingdao R3 metro line tunnel," Journal of Applied Geophysics, vol. 153, pp. 38-46, 2018.

[23] M. A. M. Ismail, T. A. Majid, C. O. Goh, S. P. Lim, and C. G. Tan, "Geological assessment for tunnel excavation under river with shallow overburden using surface site investigation data and electrical resistivity tomography," Measurement, vol. 144, pp. 260-274, 2019.

[24] A. Benedetto, F. Tosti, L. D'Amico, and F. Damico, "An overview of ground-penetrating radar signal processing techniques for road inspections," Signal Processing, vol. 132, pp. 201-209, 2017.

[25] C. Li, M.-J. Li, Y.-G. Zhao et al., "Layer recognition and thickness evaluation of tunnel lining based on ground penetrating radar measurements," Journal of Applied Geophysics, vol. 73, no. 1, pp. 45-48, 2011.

[26] S. C. Li, Z. Q. Zhou, Z. H. Ye, L. P. Li, Q. Q. Zhang, and Z. H. Xu, "Comprehensive geophysical prediction and treatment measures of karst caves in deep buried tunnel," Journal of Applied Geophysics, vol. 116, pp. 247-257, 2015.

[27] A. M. Alani and F. Tosti, "GPR applications in structural detailing of a major tunnel using different frequency antenna systems," Construction and Building Materials, vol. 158, pp. 1111-1122, 2018.

[28] F. Zhang, X. Xie, and H. Huang, "Application of ground penetrating radar in grouting evaluation for shield tunnel construction," Tunnelling and Underground Space Technology, vol. 25, no. 2, pp. 99-107, 2010.

[29] L. Wei, D. R. Magee, and A. G. Cohn, "An anomalous event detection and tracking method for a tunnel look-ahead ground prediction system," Automation in Construction, vol. 91, pp. 216-225, 2018.

[30] K. Zhao, M. Janutolo, G. Barla, and G. Chen, "3D simulation of TBM excavation in brittle rock associated with fault zones: the Brenner exploratory tunnel case," Engineering Geology, vol. 181, pp. 93-111, 2014.

[31] N. Liu, C. S. Zhang, W. J. Chu, J. Y. Wu, and P. Z. Chen, "Comprehensive inversion analysis method and application of deep buried long tunnel geo-stress field," China Journal of Highway and Transport, vol. 31, no. 10, pp. 69-78, 2018.

[32] C. Vanneschi, R. Salvini, G. Massa, S. Riccucci, and A. Borsani, "Geological 3D modeling for excavation activity in an underground marble quarry in the apuan alps (Italy)," Computers \& Geosciences, vol. 69, pp. 41-54, 2014.
[33] Z. Xiong, J. Guo, Y. Xia, H. Lu, M. Wang, and S. Shi, “A 3D multi-scale geology modeling method for tunnel engineering risk assessment," Tunnelling and Underground Space Technology, vol. 73, pp. 71-81, 2018.

[34] A. Tonini, E. Guastaldi, G. Massa, and P. Conti, "3D geomapping based on surface data for preliminary study of underground works: a case study in val Topina (Central Italy)," Engineering Geology, vol. 99, no. 1-2, pp. 61-69, 2008.

[35] L. Thum and R. De Paoli, "2D and 3D GIS-based geological and geomechanical survey during tunnel excavation," Engineering Geology, vol. 192, pp. 19-25, 2015.

[36] P.., W. Mcdowell, R. D. Barker, A. P. Butcher et al., Geophysics in Engineering Investigations, Westminster, London, UK, 2002.

[37] J. L. Davis and A. P. Annan, "Ground-penetrating radar for high-resolution mapping of soil and rock Stratigraphy1," Geophysical Prospecting, vol. 37, no. 5, pp. 531-551, 1989.

[38] E. J. Min, J. G. Shin, Y. Kim, and B. H. Lee, "Two-dimensional scanning probe driven by a solenoid-based single actuator for optical coherence tomography," Optics Letters, vol. 36, no. 11, pp. 1963-1965, 2011.

[39] J. R. Frederick, Ultrasonic Engineering, John Willey \& Sons, Inc, Hoboken, NJ, USA, 1965.

[40] G. S. Kino, Acoustic Waves: Devices, Imaging, and Analog Signal Processing, Prentice-Hall, Inc, Upper Saddle River, NJ, USA, 1987.

[41] J. A. Johnson and B. A. Barna, "The effects of surface mapping corrections with synthetic-aperture focusing techniques on ultrasonic imaging," IEEE Transactions on Sonics and Ultrasonics, vol. 30, no. 5, pp. 283-294, 1983.

[42] C. Ozdemir, S. Demirci, and E. Yigit, "Practical algorithms to focus B-scan gpr images: theory and application to real data," Progress In Electromagnetics Research B, vol. 6, pp. 109-122, 2008. 